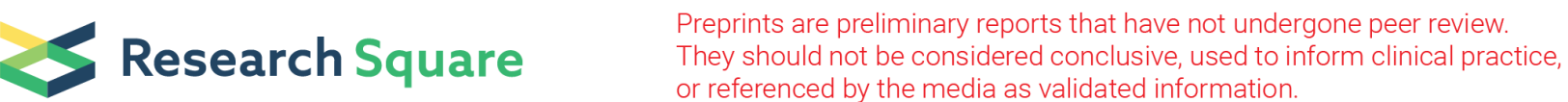

\section{Impaired odor identification ability and olfactory hedonic capacity in children with elevated autistic traits}

\section{Gao-jie Huang}

Southern Medical University

\section{Yi-qi Qiu}

Southern Medical University

Shao-zhen Tan

Guangzhou Social Welfare Instituate

Qian-wen Ma

Southern Medical University

Laiquan Zou ( $\nabla$ zoulq@smu.edu.cn )

Southern Medical University https://orcid.org/0000-0003-4948-036X

\section{Research}

Keywords: adolescent, autistic trait, children, olfactory hedonic capacity, odor identification

Posted Date: December 2nd, 2020

DOI: https://doi.org/10.21203/rs.3.rs-118795/v1

License: (c) (1) This work is licensed under a Creative Commons Attribution 4.0 International License. Read Full License 


\section{Abstract}

Background: Atypical olfactory function in autism spectrum disorder has been documented in numerous studies, but little is known about its occurrence in individuals with autistic traits. The aim of the current study was to investigate odor identification ability and olfactory hedonic capacity in children with autistic traits.

Methods: The study included 91 children in a high autistic trait group, and 128 children in a low autistic trait group, as determined based on Short Autism Spectrum Quotient scores. The Universal Sniff (U-Sniff) test was used to measure odor identification ability and olfactory hedonic capacity. Olfactory hedonic capacity was also measured using the child's version of the Chemosensory Pleasure Scale (CPS-C).

Results: Children in the high autistic trait group exhibited significantly impaired odor identification and olfactory hedonic capacity measured by CPS-C than those in the low autistic trait group, but there was no significant difference in olfactory hedonic capacity measured by U-Sniff between the two groups. Impaired odor identification was significantly correlated with olfactory hedonic capacity measured by CPS-C.

Limitations: Only a self-reporting questionnaire was used to screen children for autistic trait. The combination of a self-reporting scale and diagnosis by a clinical expert would result in more accurate screening of individuals for autistic trait.

Conclusions: Children with high autistic trait exhibited poorer odor identification ability and olfactory trait hedonic capacity than children without high autistic trait. These results pertaining to odor identification and olfactory hedonic capacity may be the endophenotypic markers for autism spectrum disorder.

\section{Introduction}

Autism spectrum disorder (ASD) encompasses a variable group of neurodevelopmental disorders characterized by social and communication impairments as well as repetitive behaviors (1). Most individuals with ASD exhibit atypical sensory capacity in childhood (2), which has been included in the DSM-5 diagnostic criteria for ASD (1). Most evidence on altered sensory processing is based on visual and auditory processing in people with ASD (3-6). Aberrant olfactory function in people with ASD has also been demonstrated in numerous studies, and the most prominent manifestation of it is odor identification (7-12). Some heterogeneous results have been reported however, and in some studies odor identification was not significantly impaired in people with ASD (13-15). Inconsistent methodology may have contributed to the heterogeneous results that have been reported (16).

The University of Pennsylvania Smell Identification Test (UPSIT) (17) and the "Sniffin' Sticks" test (18) are most commonly used to measure odor identification, but the odors they utilize may be unfamiliar to children (19). In addition, descriptors in the UPSIT and the Sniffin' Sticks test are usually presented in writing, which is not feasible to use to measure odor identification in children with ASD because of their 
delayed speech and communication impairment (8). Schriever, Agosin (19) developed the Universal Sniff (U-Sniff) test to measure odor identification in children, in which the descriptor of each odor is presented both photographically and in writing. In addition, the U-Sniff odors were selected by children from different countries, and the test has exhibited good reliability and validity in children from different countries $(19,20)$. Therefore, the USniff test was used to measure odor identification in the current study, to reduce the additional effects of speech and verbal labeling.

Hedonic capacity, which refers to the ability to experience pleasure, is considered a crucial index for understanding the core impairments in ASD-social and affective deficits $(21,22)$. People with ASD exhibit significant impairment in physical and social hedonic capacity (21). The overlapping brain areas associated with olfaction and emotional processing suggest that olfaction may have an unusual impact on emotion perception (23). In previous studies people with ASD have exhibited diminished odor hedonic discrimination and judgement capacity $(9,12)$.

Autistic traits were continuously distributed in the general population (24), which would benefit heritable ASD marker research. Individuals with so-called "autistic trait", also referred as the broader autism phenotype, are a group of people who have autistic traits but their severity is subclinical $(24,25)$. Therefore, it is reasonable to hypothesize that individuals with autistic trait also have odor identification impairment and olfactory anhedonia. To our knowledge no study has examined odor identification in individuals with autistic trait. Novacek, Gooding (22) reported that young adults with autistic trait exhibited significantly lower social pleasure, but olfactory hedonic capacity in children/adolescents with autistic trait is understudied.

The aim of the current study was to investigate odor identification ability and olfactory hedonic capacity in children with autistic trait. We hypothesized that children with autistic trait would exhibit reduced odor identification ability and olfactory hedonic capacity.

\section{Methods}

\subsection{Participants}

The study included 91 children with high autistic trait as determined via Short Autism Spectrum Quotient (AQ-10) scores (26), 35 boys and 56 girls, with a mean age of $13.01 \pm 2.93$ years (range $9-17$ years). The AQ-10 was also used to identify 128 children with low autistic trait scores, 50 boys and 78 girls, who had a mean age of $13.43 \pm 2.71$ years (range $9-17$ years). The children were recruited from several schools via convenient sampling. A total of 415 students were initially administered the AQ-10, and those with scores $\geq 6$ were assigned to the high autistic trait (high AQ) group, and those with scores $\leq 3$ were assigned to the low autistic trait (low AQ) group (27). Participants were excluded if they had had a bad cold in the past week, or if they had a history of nasal diseases, brain injury, or any neuropsychiatric disorders as determined via a personal interview with a trained interviewer. The study was approved by the Ethics Committee of Southern Medical University, and conducted in accordance with the principles of 
the Declaration of Helsinki. Informed consent was obtained from teachers and the children's legal guardians.

\subsection{Measures}

\subsubsection{The U-Sniff test}

Odor identification ability and olfactory hedonic capacity were assessed using the USniff test (19). The USniff test utilizes 12 pen-like "Sniffin' Sticks", which emit apple, banana, butter, coffee, cut grass, fish, flower, lemon, onion, orange, peach, and strawberry odors. It has previously exhibited good reliability and validity $(19,20)$, and in a study conducted in China its test-retest reliability was $0.69(20)$.

The test was conducted in a quiet and well-ventilated room. Before odor presentation, the descriptors were showed to the participant and read aloud by the examiner. One odor at a time was birhinally presented to the participant for 3 seconds. The participant made a forced choice from four given descriptors, which were presented in picture and textual forms. The participant was asked to smell the odor again if they were not sure of their choice. In addition, participants were asked to rate the perceived odor pleasantness from 1 ("very much dislike this odor") to 9 ("very much like this odor") based on their subjective feelings. Odor identification ability was scored as the sum of correct odor choices. The total odor identification score for each participant ranged from $0-12$, and higher scores represented better odor identification ability. The sum of the odor pleasantness ratings was used to quantify olfactory hedonic capacity, and higher scores represented better olfactory hedonic capacity.

\subsubsection{The child's version of the Chemosensory Pleasure Scale}

Olfactory hedonic capacity was also assessed via the child's version of the Chemosensory Pleasure Scale (CPS-C), in which the expression of the standard Chemosensory Pleasure Scale (28) items is modified to facilitate understanding by children. As with the standard Chemosensory Pleasure Scale, the CPS-C consists of 12 items encompassing three broader factors, "Food", "Imagination", and "Nature", which respectively relate to hedonic capacity while eating, anticipating food, and smelling natural scents. Participants were asked to respond using a 6-point Likert scale ranging from 1 ("not at all true for me") to 6 ("very true for me"). The sum of scores from the CPS-C items indicated the participant's olfactory hedonic capacity, and higher scores represented better olfactory hedonic capacity. The test-retest reliability of the CPS-C was 0.718 .

\subsubsection{The Short Autism Spectrum Quotient}

Autistic trait was assessed using the Short Autism Spectrum Quotient (AQ-10) (26), which contains the 10 screening items from the 50-item Autism Spectrum Quotient (29) with the highest sensitivity and specificity. It has been confirmed that the AQ-10 is a useful screening instrument for autistic trait and ASD $(26,30)$. There are child, adolescent, and adult versions of the AQ-10, which were used to measure autistic traits in the participants in the current study based on their ages. The participants who aged from 58 years and 9-11 years used the child version of AQ-10 and the participants who aged 12-14 years and 15-17 years respectively used the adolescent version and adult version of AQ-10. The participants were asked to choose the degree to which they agreed or disagreed with the statements in the AQ-10. Each 
item has four alternative choices: strongly agree, slightly agree, slightly disagree, and strongly disagree. In the current study the response to each item was recorded as " 1 " if the choice was "strongly agree" or "slightly agree", and " 0 " if it was "slightly disagree" or "strongly disagree". All scores were summed, and higher scores represented more severe autistic trait symptomatology.

\subsection{Data analysis}

Statistical analysis was performed using SPSS 25.0 (SPSS Inc, Chicago, IL, USA). The $t$-test for independent-samples or the chi-square test were used to assess the significance of differences in age or sex between the high AQ group and the low $A Q$ group. Group differences between the high AQ group and the low AQ group assessed using the $t$-test for independent samples. In addition, the effect size estimates of the Independent $t$-test were calculated using Cohen $d$ and phi estimate was used to measure the effect size of chi-square test. The Shapiro-Wilk normality test was used to assess the normality of data distributions in each group. Relationships between odor identification score and CPS-C total or subscale scores were assessed in each group. Pearson's correlations were calculated if the data were normally distributed, and Spearman's correlations were calculated if the data were not normally distributed.

\section{Results}

Age did not differ significantly in the high AQ group and the low AQ group $(t=1.089, p=0.277, d=0.15)$, and neither did sex $\left(\square^{2}=0.008, p=0.928, \Phi=0.006\right)$. Odor identification scores were significantly lower in the high AQ group ( $t=2.43, p=0.016, d=0.34)$, as were total CPS-C scores $(t=3.71, p<0.001, d=0.50)$ and all CPS-C subscale scores (CPS-C Nature: $t=2.81, p=0.006, d=0.39$; CPS-C Food: $t=2.90, p=0.004$, $d=0.39$; CPSC imagination: $t=3.33, p=0.001, d=0.46$ ) (Fig. 1). There was no significant difference in odor pleasantness ratings between the two groups $(t=0.65, p=0.520, d=0.09)$ (Table 1, Fig. 2). 
Table 1

Olfactory test performance and olfactory hedonic capacity ( $x \pm s)$.

\begin{tabular}{|c|c|c|c|c|c|}
\hline & Low AQ group & High AQ group & $t$ & $p$ & $d$ \\
\hline & $(n=128)$ & $(n=91)$ & & & \\
\hline Odor identification score & $11.00 \pm 1.47$ & $10.42 \pm 1.92$ & 2.43 & 0.016 & 0.34 \\
\hline Odor pleasantness ratings & $68.55 \pm 12.74$ & $67.38 \pm 13.91$ & 0.65 & 0.520 & 0.09 \\
\hline CPS-C Nature score & $14.23 \pm 2.44$ & $13.14 \pm 3.06$ & 2.81 & 0.006 & 0.39 \\
\hline CPS-C Food score & $24.60 \pm 4.60$ & $22.68 \pm 5.15$ & 2.90 & 0.004 & 0.39 \\
\hline CPS-C Imagination score & $17.24 \pm 4.21$ & $15.37 \pm 3.94$ & 3.33 & 0.001 & 0.46 \\
\hline CPS-C total score & $56.07 \pm 9.00$ & $51.20 \pm 10.33$ & 3.71 & $<0.001$ & 0.50 \\
\hline
\end{tabular}

In the high AQ group odor identification score was significantly positively correlated with odor pleasantness ratings $(r=0.288, p<0.01)$, total CPS-C score $(r=0.477, p<0.01)$, and all CPS-C subscale scores (CPS-C Nature $r=0.371, p<0.01$; CPS-C Food $r=0.407, p<0.01$; CPS-C Imagination $r=0.391, p<$ $0.01)$. In the low $A Q$ group odor identification score was not significantly associated with total CPS-C score or any CPS-C subscale scores except CPS-C Nature $(r=0.182, p<0.05)$.

\section{Discussion}

To our knowledge the current study is the first to directly measure odor identification and olfactory hedonic capacity in children with autistic trait. It is also the first study in which psychophysical measurement and self-reporting scales were both used to assess olfactory hedonic capacity. Children with autistic trait exhibit impaired odor identification. Interestingly, in the present study there was a pattern of impaired olfactory hedonic capacity measured by CPS-C, but intact olfactory hedonic capacity measured by U-Sniff. Children in the high AQ group were worse than those in the low HQ group at odor identification and olfactory hedonic capacity as measured by the CPSC, but the groups did not differ significantly in olfactory hedonic capacity as measured by the U-Sniff test. There was also a significant association between odor identification and olfactory hedonic capacity measured by the CPS-C in the high $A Q$ group, but not in the low $A Q$ group.

In the current study children in the high AQ group exhibited impaired odor identification, which is not consistent with Dudova, Vodicka (15) or Brewer, Brereton (14), in which odor identification did not differ significantly in children/adolescents with ASD and healthy controls. A possible reason for the inconsistent results is the limitations of the only previously available odor identification tools, which are restricted by speech and communication capacity. The results of the current study are consistent with 
previous studies in which abnormal odor identification was detected in children/adolescents with ASD (2, $7,10)$, suggesting that attenuated odor identification may be an endophenotypic marker of children/adolescents with high autistic trait.

In the present study lower olfactory hedonic capacity was evident using the CPS-C, but not the U-Sniff test. This is consistent with a recent meta-analysis in which laboratory-based methods did not detect impaired olfactory hedonic capacity in people with ASD (31). In a previous report it was suggested that hedonic capacity was composed of trait hedonic capacity and state hedonic capacity (32). Trait hedonic capacity was defined as "non-current" hedonic capacity assessed via interviewing or self-reporting scales, and state hedonic capacity was defined as "current" hedonic capacity assessed via laboratorybased measures. In the present study children with high autistic trait exhibited significantly impaired olfactory trait hedonic capacity and intact olfactory state hedonic capacity.

Robinson and Clore (33) proposed the accessibility model of emotional self-reporting, in which people mainly rely on experimental knowledge when reporting their current feelings, whereas non-current feelings are based on their semantic emotional knowledge, including episodic memory, beliefs about the particular situation, and their general beliefs about themselves $(33,34)$. "Non-current" hedonic capacity includes prospective hedonic capacity for the future and retrospective hedonic capacity for the past. Long-term memories related to a specific situation play a key role in retrospective hedonic experience (34). In a previous study people with ASD exhibited impaired long-term memory, especially detailed memory (35). Like people with ASD, people with high autistic trait also exhibit lower neuropsychological performance (i.e., memory, attention, and learning) (36). Thus, in such individuals impaired long-term memoryespecially episodic memory-may influence reported olfactory retrospective hedonic capacity.

More complex cognitive skills are required for prospective hedonic experience, including working memory, imagination, and reflection (37). Strauss (34) surmised that it was essential to generate a mental representation to elicit a hedonic reaction in the present when measuring prospective hedonic capacity, and the mental representation mainly relied on the ability of working memory and the peaking experience of the situation in the past. Notably however, in people with ASD or autistic trait the mental representation may be too abbreviated or it may be difficult to extract the relative peaking experience in the past because of impairment of working memory and long-term memory $(35,38-40)$. Thus, in the present study children in the high $A Q$ group reported lower olfactory prospective hedonic capacity than those in the low $A Q$ group. Therefore, lower olfactory trait hedonic capacity may be an endophenotypic marker of ASD, which may reflect the impaired cognitive function associated with the disorder.

In the current study there was a significant association between odor identification capacity and olfactory hedonic capacity as measured by the CPS-C in children in the high AQ group, but not those in the low AQ group. This further suggests that in children with high autistic trait attenuated odor identification may be affected by impaired emotional and cognitive function, but not current hedonic capacity. Odor identification processing is performed at the central level, including the orbitofrontal cortex, hippocampus, 
insula, and prefrontal cortex (23). This brain area is associated with emotion processing, social control, and working memory, which are characteristically involved in ASD (10).

It has previously been reported that hedonic capacity played a key role in understanding social and affective disorders in people with ASD $(21,22)$, and that odor identification ability was significantly affected by cognitive function (i.e., semantic memory, executive function, and episodic memory) (41) at the behavioral level. Accordingly, abnormally low odor identification ability may be a marker of impaired emotional and cognitive function in people with ASD.

\section{Limitations}

The current study had some limitations. Only a self-reporting questionnaire was used to screen children for autistic trait. The combination of a self-reporting scale and diagnosis by a clinical expert would result in more accurate screening of individuals for autistic trait. Additionally, although in the high AQ group there was significantly lower olfactory trait hedonic capacity but not significantly lower olfactory state hedonic capacity, future studies are needed to explore the neural mechanism of this pattern of olfactory hedonic anhedonia.

\section{Conclusions}

In the current study children with high autistic trait exhibited poorer odor identification ability and olfactory trait hedonic capacity than children without high autistic trait. Poorer odor identification ability was also associated with olfactory hedonic capacity in children with high autistic trait. This knowledge about odor identification and olfactory hedonic capacity may suggest that impaired odor identification and olfactory hedonic capacity are the endophenotypic markers for ASD.

\section{Abbreviations}

ASD: Autism Spectrum Disorder

U-Sniff: Universal Sniff

CPS: Chemosensory Pleasure Scale

CPS-C: the child of Chemosensory Pleasure Scale

AQ-10: Short Autism Spectrum Quotient

AQ: Autism Spectrum Quotient

\section{Declarations}

\section{Ethics approval and consent to participate}


The study was approved by the Ethics Committee of Southern Medical University, and conducted in accordance with the principles of the Declaration of Helsinki. Informed consent was obtained from teachers and the children's legal guardians

\section{Consent for publication}

Not applicable

\section{Availability of data and materials}

The datasets used and analyzed during the current study are available in the corresponding author on reasonable request.

\section{Competing interests}

The authors declare that they have no competing interests.

\section{Funding}

This study was supported by the National Natural Science Foundation of China (grant number 31700963), the Natural Science Foundation of Guangdong Province, China (grant number 2019A1515012135), and the Medical Science and Technology Foundation of Guangdong Province, China (grant number A2019192). These funding agents had no further role in the study design; in the collection, analysis and interpretation of the data; in the writing of the manuscript; and in the decision to submit the paper for publication.

\section{Authors' contributions}

GJH collected and analyzed the data, and wrote the first draft of the manuscript. YQQ collected, analyzed and interpreted the data. SZT interpreted the data and gave the comment to the first draft of the manuscript. QWM and LQZ generated the idea, designed the study, interpreted the data, and wrote the first draft of the manuscript. All authors have read and approved the final manuscript.

\section{Acknowledgements}

The authors would like to acknowledge those participants taking part in this study and the support of the funding sources.

\section{Authors' information}

Affiliations

Chemical Senses and Mental Health Laboratory, Department of Psychology, School of Public Health, Southern Medical University, Guangzhou, Guangdong, China 
Gao-jie Huang, Yi-qi Qiu, Qian-wen Ma, Lai-quan Zou

Department of Psychiatry, Zhujiang Hospital, Southern Medical University, Guangzhou, Guangdong, China Lai-quan Zou

Guangzhou Social Welfare Institute, Guangzhou, Guangdong, China

Shao-zhen Tan

Corresponding authors

Correspondence to Qian-wen Ma or Lai-quan Zou.

\section{References}

1. American Psychiatric Association. Diagnostic and statistical manual of mental disorders (DSM-5®). Washington DC: American Psychiatric Pub; 2013.

2. Baum SH, Stevenson RA, Wallace MT. Behavioral, perceptual, and neural alterations in sensory and multisensory function in autism spectrum disorder. Prog Neurobiol. 2015;134:140-60.

3. Hilton CL, Harper JD, Kueker RH, Lang AR, Abbacchi AM, Todorov A, et al. Sensory responsiveness as a predictor of social severity in children with high functioning autism spectrum disorders. Journal of autism and developmental disorders. 2010;40(8):937-45.

4. Tan Y-H, Xi C-Y, Jiang S-P, Shi B-X, Wang L-B, Wang L. Auditory abnormalities in children with autism. Open Journal of Psychiatry. 2012;02(01):33-7.

5. Pierce K, Marinero S, Hazin R, McKenna B, Barnes CC, Malige A. Eye Tracking Reveals Abnormal Visual Preference for Geometric Images as an Early Biomarker of an Autism Spectrum Disorder Subtype Associated With Increased Symptom Severity. Biological psychiatry. 2016;79(8):657-66.

6. Haigh SM. Variable sensory perception in autism. European Journal of Neuroscience. 2018;47(6):602-9.

7. Bennetto L, Kuschner ES, Hyman SL. Olfaction and Taste Processing in Autism. Biological psychiatry. 2007;62(9):1015-21.

8. Galle SA, Courchesne V, Mottron L, Frasnelli J. Olfaction in the autism spectrum. Perception. 2013;42(3):341-55.

9. Luisier AC, Petitpierre G, Ferdenzi C, Clerc Berod A, Giboreau A, Rouby C, et al. Odor Perception in Children with Autism Spectrum Disorder and its Relationship to Food Neophobia. Front Psychol. 2015;6:1830.

10. Muratori F, Tonacci A, Billeci L, Catalucci T, Igliozzi R, Calderoni S, et al. Olfactory Processing in Male Children with Autism: Atypical Odor Threshold and Identification. Journal of autism and developmental disorders. 2017;47(10):3243-51. 
11. Suzuki Y, Critchley HD, Rowe A, Howlin P, Murphy DGM. Impaired olfactory identification in Asperger's syndrome. The Journal of neuropsychiatry and clinical neurosciences. 2003;15(1):105-7.

12. Wicker B, Monfardini E, Royet JP. Olfactory processing in adults with autism spectrum disorders. Mol Autism. 2016;7:4.

13. Addo RN, Wiens S, Nord M, Larsson M. Olfactory Functions in Adults With Autism Spectrum Disorders. Perception. 2017;46(3-4):530-7.

14. Brewer WJ, Brereton A, Tonge BJ. Dissociation of age and ability on a visual analogue of the University of Pennsylvania Smell Identification Test in children with autism. Research in Autism Spectrum Disorders. 2008;2(4):612-20.

15. Dudova I, Vodicka J, Havlovicova M, Sedlacek Z, Urbanek T, Hrdlicka M. Odor detection threshold, but not odor identification, is impaired in children with autism. Eur Child Adolesc Psychiatry. 2011;20(7):333-40.

16. Boudjarane MA, Grandgeorge M, Marianowski R, Misery L, Lemonnier E. Perception of odors and tastes in autism spectrum disorders: A systematic review of assessments. Autism Res. 2017;10(6):1045-57.

17. Doty RL, Shaman P, Dann M. Development of the University of Pennsylvania Smell Identification Test: a standardized microencapsulated test of olfactory function. Physiol Behav. 1984;32(3):489502.

18. Hummel T, Sekinger B, Wolf SR, Pauli E, Kobal G. 'Sniffin' sticks': olfactory performance assessed by the combined testing of odor identification, odor discrimination and olfactory threshold. Chem Senses. 1997;22(1):39-52.

19. Schriever VA, Agosin E, Altundag A, Avni H, Cao Van H, Cornejo C, et al. Development of an International Odor Identification Test for Children: The Universal Sniff Test. J Pediatr. 2018;198:265$72 \mathrm{e} 3$.

20. Zou LQ, Dworschak A, Alizadeh R, Kamrava SK, Alwashahi M, Bock MA, et al. "U-Sniff" - the international odor identification test for children: an extension of its normative database and study of global reliability. Rhinology. 2020.

21. Berthoz S, Lalanne C, Crane L, Hill EL. Investigating emotional impairments in adults with autism spectrum disorders and the broader autism phenotype. Psychiatry Res. 2013;208(3):257-64.

22. Novacek DM, Gooding DC, Pflum MJ. Hedonic Capacity in the Broader Autism Phenotype: Should Social Anhedonia Be Considered a Characteristic Feature? Front Psychol. 2016;7:666.

23. Gottfried JA. Smell: central nervous processing. Advances in oto-rhino-laryngology. 2006;63:44-69.

24. Constantino JN, Todd RD. Autistic traits in the general population: a twin study. Archives of general psychiatry. 2003;60(5):524-30.

25. Ruzich E, Allison C, Smith P, Watson P, Auyeung B, Ring H, et al. Measuring autistic traits in the general population: a systematic review of the Autism-Spectrum Quotient (AQ) in a nonclinical population sample of 6,900 typical adult males and females. Mol Autism. 2015;6:2. 
26. Allison C, Auyeung B, Baron-Cohen S. Toward brief "Red Flags" for autism screening: The Short Autism Spectrum Quotient and the Short Quantitative Checklist for Autism in toddlers in 1,000 cases and 3,000 controls [corrected]. Journal of the American Academy of Child and Adolescent Psychiatry. 2012;51(2):202-12.e7.

27. Nijhof $A D$, Brass $M$, Wiersema JR. Spontaneous mentalizing in neurotypicals scoring high versus low on symptomatology of autism spectrum disorder. Psychiatry Res. 2017;258:15-20.

28. Zhao JB, Wang YL, Ma QW, Zhao JB, Zhang XY, Zou LQ. The Chemosensory Pleasure Scale: A New Assessment for Measuring Hedonic Smell and Taste Capacities. Chem Senses. 2019;44(7):457-64.

29. Baron-Cohen S, Wheelwright S, Skinner R, Martin J, Clubley E. The autism-spectrum quotient (AQ): evidence from Asperger syndrome/high-functioning autism, males and females, scientists and mathematicians. Journal of autism and developmental disorders. 2001;31(1):5-17.

30. Booth T, Murray AL, McKenzie K, Kuenssberg R, O'Donnell M, Burnett $H$. Brief report: an evaluation of the AQ-10 as a brief screening instrument for ASD in adults. Journal of autism and developmental disorders. 2013;43(12):2997-3000.

31. Crow AJD, Janssen JM, Vickers KL, Parish-Morris J, Moberg PJ, Roalf DR. Olfactory Dysfunction in Neurodevelopmental Disorders: A Meta-analytic Review of Autism Spectrum Disorders, Attention Deficit/Hyperactivity Disorder and Obsessive-Compulsive Disorder. Journal of autism and developmental disorders. 2020.

32. Yan C, Cao Y, Zhang Y, Song LL, Cheung EF, Chan RC. Trait and state positive emotional experience in schizophrenia: a meta-analysis. PLoS One. 2012;7(7):e40672.

33. Robinson MD, Clore GL. Belief and feeling: evidence for an accessibility model of emotional selfreport. Psychological bulletin. 2002;128(6):934-60.

34. Strauss GP. The emotion paradox of anhedonia in schizophrenia: or is it? Schizophr Bull. 2013;39(2):247-50.

35. Williams DL, Minshew NJ, Goldstein G, Mazefsky CA. Long-term memory in older children/adolescents and adults with autism spectrum disorder. Autism Res. 2017;10(9):1523-32.

36. Hyseni F, Blanken LME, Muetzel R, Verhulst FC, Tiemeier H, White T. Autistic traits and neuropsychological performance in 6- to-10-year-old children: a population-based study. Child Neuropsychol. 2019;25(3):352-69.

37. Kring AM, Caponigro JM. Emotion in Schizophrenia: Where Feeling Meets Thinking. Current directions in psychological science. 2010;19(4):255-9.

38. Boucher J, Mayes A, Bigham S. Memory in autistic spectrum disorder. Psychological bulletin. 2012;138(3):458-96.

39. Richmond LL, Thorpe M, Berryhill ME, Klugman J, Olson IR. Individual differences in autistic trait load in the general population predict visual working memory performance. Quarterly journal of experimental psychology (2006). 2013;66(6):1182-95.

40. Williams DL, Goldstein G, Minshew NJ. Impaired memory for faces and social scenes in autism: clinical implications of memory dysfunction. Archives of clinical neuropsychology : the official 
journal of the National Academy of Neuropsychologists. 2005;20(1):1-15.

41. Hedner M, Larsson M, Arnold N, Zucco GM, Hummel T. Cognitive factors in odor detection, odor discrimination, and odor identification tasks. Journal of clinical and experimental neuropsychology. 2010;32(10):1062-7.

Figures

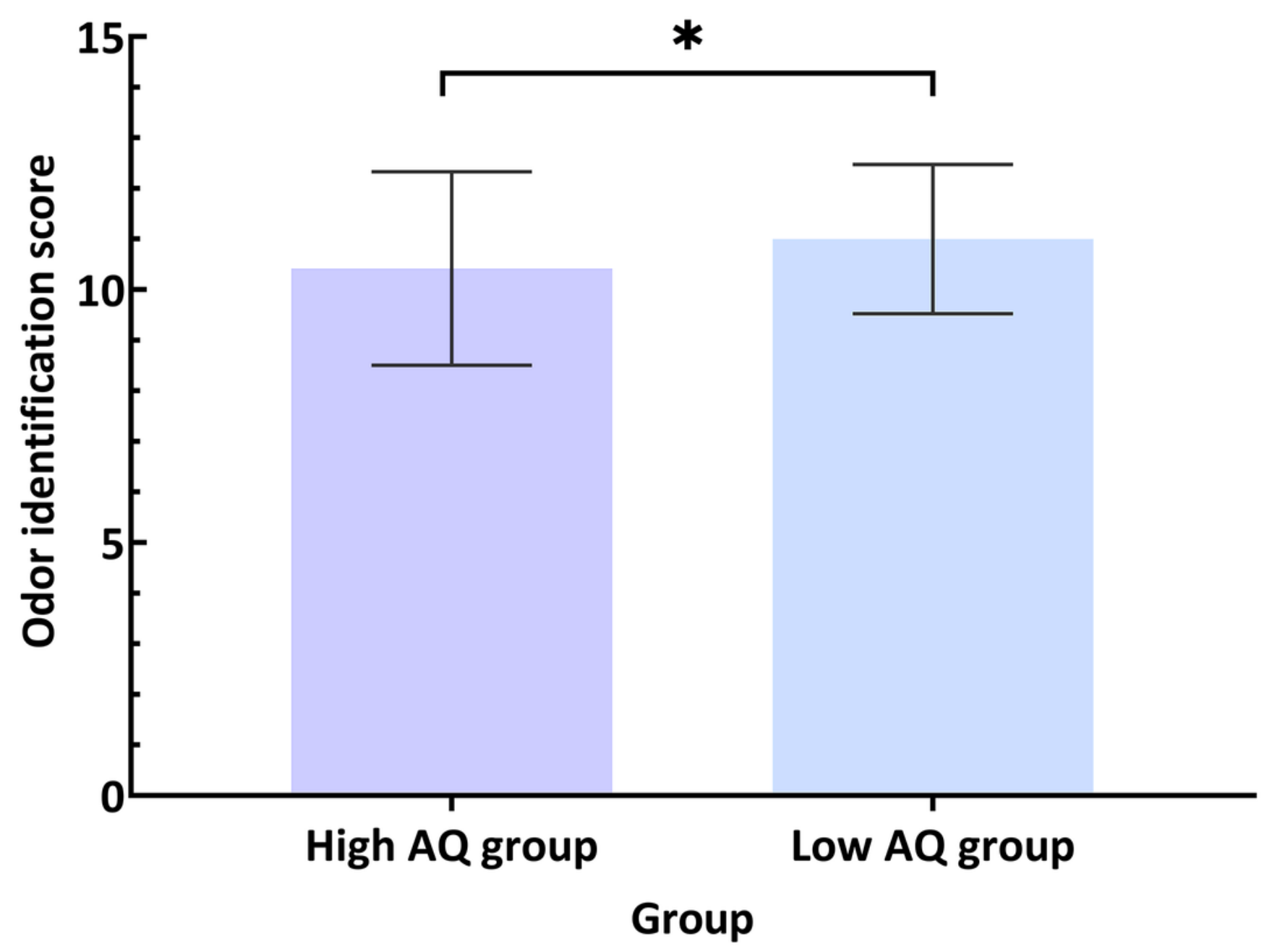

Figure 1

Odor identification scores in the high autism quotient group and the low autism quotient group. Note. AQ, autism quotient. ${ }^{*} p<0.01$ 


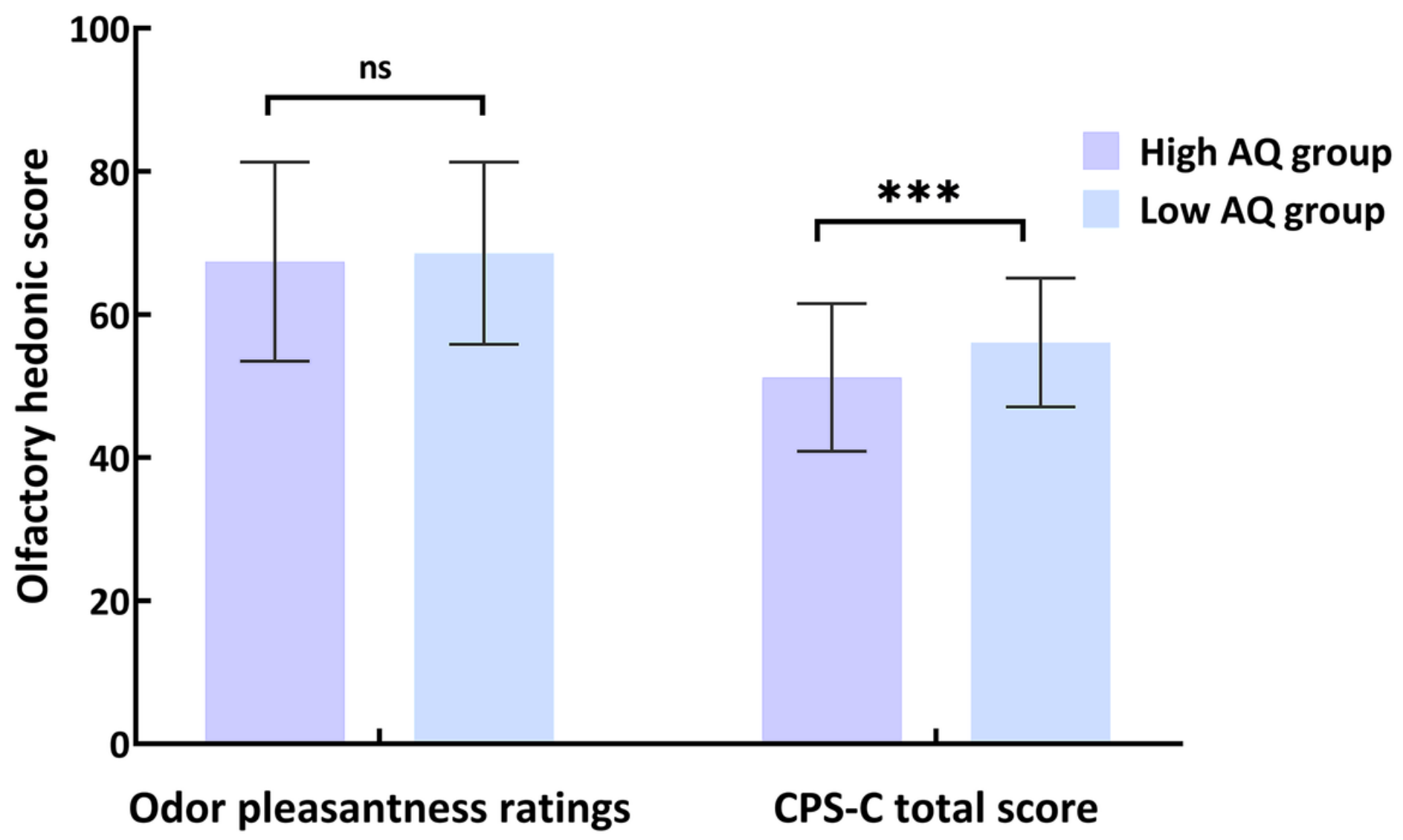

Figure 2

Olfactory hedonic scores in the high autism quotient group and the low autism quotient group. Note. ns, non-significant difference; $A Q$, autism quotient; $C P S-C$, child's version of the Chemosensory Pleasure Scale. ${ }^{* \star *} p<0.001$ 